\title{
ERROR IDENTIFICATION AND COMPENSATION IN LARGE MANIPULATORS WITH APPLICATION IN CANCER PROTON THERAPY
}

\author{
Meggiolaro, M.A.*
}

\author{
S. Dubowsky ${ }^{\dagger}$
}

\author{
C. Mavroidis ${ }^{\ddagger}$ \\ *PUC-Rio, Dept. of Mechanical Engineering - Rua Marquês de São Vicente, 225 - Gávea, Rio de Janeiro, RJ \\ ${ }^{\dagger}$ MIT, Dept. of Mechanical Engineering, 77 Massachusetts Ave, Cambridge, MA 02139, USA \\ ${ }^{\ddagger}$ Rutgers University, Dept. of Mechanical and Aerospace Engineering, 98 Brett Road, Piscataway, NJ 08854, USA
}

\begin{abstract}
Important robotic tasks could be effectively performed by powerful and accurate manipulators. However, high accuracy is generally difficult to obtain in large manipulators capable of producing high forces due to system elastic and geometric distortions. A method is presented to identify the sources of end-effector positioning errors in large manipulators using experimentally measured data. The method does not require explicit structural modeling of the system. Both geometric and elastic deformation positioning errors are identified. These error sources are used to predict, and compensate for, end-point errors as a function of configuration and measured forces, improving the system absolute accuracy. The method is applied to a large high-accuracy medical robot. Experimental results show that the method is able to effectively correct for the system errors.
\end{abstract}

KEYWORDS: Robot calibration, Flexible arms, Medical systems.

\section{RESUMO}

Importantes tarefas robóticas poderiam ser eficientemente

\footnotetext{
Artigo submetido em 02/12/02

1a. Revisão em 12/05/03

Aceito sob recomendação do Ed. Assoc. Prof. Paulo E. Miyagi
}

executadas por manipuladores potentes e precisos. No entanto, alta precisão absoluta é geralmente difícil de ser obtida em grandes manipuladores capazes de produzir forças de alta intensidade, devido às distorções elásticas e geométricas do sistema. Um método é apresentado para identificar, através de medições indiretas, as fontes de erro de posicionamento da extremidade de grandes manipuladores. O método não requer uma modelagem estrutural explícita do sistema. Ambos os erros geométricos e elásticos de posicionamento são identificados. Esses valores são usados para predizer e compensar os erros da extremidade do robô em função da configuração e de forças medidas, melhorando a precisão absoluta do sistema. O método é aplicado a um grande robô médico de alta precisão. Resultados experimentais demonstram que o método é capaz de corrigir eficientemente os erros de posicionamento do sistema.

PALAVRAS-CHAVE: Calibragem de robôs, manipuladores flexíveis, sistemas médicos.

\section{INTRODUCTION}

Large robot manipulators are needed in field, service and medical applications to perform high accuracy tasks. Examples are manipulators that perform decontamination tasks in nuclear sites, space manipulators such as the Special Purpose Dexterous Manipulator (SPDM) and manipulators for medi- 
cal treatment (Vaillancourt et al. 1994; Flanz 1996; Hamel et al. 1997). In these applications, a large robotic system is often needed to have very fine precision. Its accuracy specifications may be very small fractions of its size. Achieving such high accuracy is difficult because of the manipulator's size and its need to carry heavy payloads. Further, many tasks, such as space applications, require systems to be lightweight, and thus structural deformation errors can be large.

In such systems, two principal error sources create significant end-effector errors. The first is kinematic errors due to the non-ideal geometry of the links and joints of manipulators, such as errors due to machining tolerances. These errors are often called geometric errors. Task constraints often make it impossible to use direct end-point sensing in a closed-loop control scheme to compensate for these errors. Therefore, there is a need for model-based robot calibration.

The second error source that can limit the absolute accuracy of a large manipulator is the elastic errors due to the distortion of a manipulator's mechanical components under large task loads or even its own weight. Classical error compensation methods cannot correct the errors in large systems with significant elastic deformations, because they do not explicitly consider the effects of task forces and structural compliance. Methods have been developed to deal with this problem (Drouet et al. 1998), however they depend upon lenghty analytical models of the manipulator structural properties.

In this work a method that compensates for the position and orientation errors caused by geometric and elastic errors in large manipulators is discussed. The method, called here Geometric and Elastic Error Compensation (GEC), explicitly considers the task load dependency of the errors, modeling both deformation and more classical geometric errors in a unified and simplified manner. A set of experimentally measured positions and orientations of the robot end-effector and measurements of the payload wrench are used to calculate the robot "generalized" errors without using an explicit manipulator elastic model. Without the need of an explicit elastic model, it is feasible to completely automate the analytical derivation of the required identification matrices. Generalized are called the errors that characterize the relative position and orientation of frames defined at the manipulator links. They are determined from measured data as a function of the system configuration and the task wrench. Knowing these generalized errors the manipulator end-effector errors are used to compensate for robot errors at any configuration. In the GEC method each generalized error parameter can be represented as a function of only a few of the system variables. As a result, the number of measurements required to characterize the system is significantly smaller than expected.

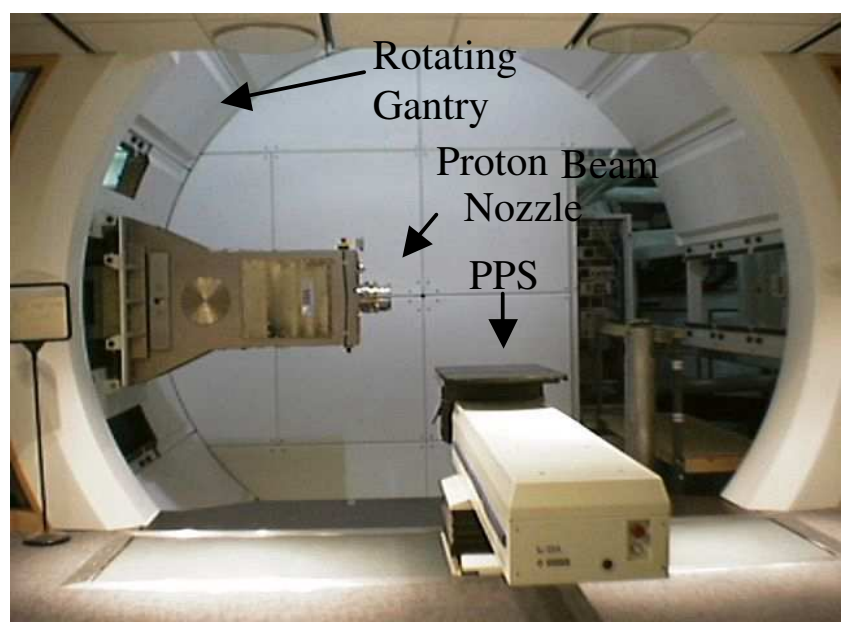

Figure 1: The PPS and the Gantry

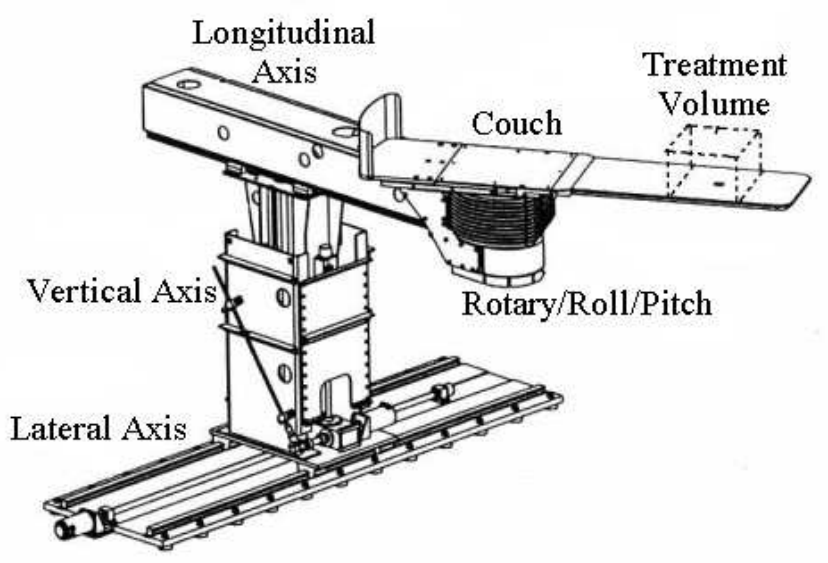

Figure 2: The Patient Positioning System

The method is applied to an important medical application of large manipulator systems. The manipulator is used as a high accuracy robotic patient positioning system in a radiation therapy research facility constructed at the Massachusetts General Hospital (MGH), the Northeast Proton Therapy Center (NPTC) (Flanz 1996). The robotic patient positioning system (PPS) places a patient in a high energy proton beam delivered from a proton nozzle carried by a rotating gantry structure (see Fig. 1). The PPS is a six degree of freedom manipulator that covers a large workspace of more than $4 \mathrm{~m}$ in radius while carrying patients weighing as much as $300 \mathrm{lbs}$ (see Fig. 2). Patients are immobilized on a "couch" attached to the PPS end-effector. The PPS, combined with the rotating gantry that carries the proton beam, enables the beam to enter the patient from any direction, while avoiding the gantry structure. Hence flexibility offered by robotic technology is needed. 
The required absolute positioning accuracy of the PPS is 0.5 $\mathrm{mm}$. This accuracy is critical as larger errors may be dangerous to the patient (Rabinowitz 1985). The required accuracy is roughly $10^{-4}$ of the nominal dimension of system workspace. This is a greater relative accuracy than most industrial manipulators. In addition, FEM studies and experimental results show that with a changing payload (between 1 and 300 pounds) and changing configuration the end-effector errors due to elastic deformations and geometric errors are of the order of $6-8 \mathrm{~mm}$. Hence the accuracy is 12 to 16 times the system specification (Mavroidis et al. 1997). However, since the repeatability error of the PPS, defined here as how well the system returns to certain arbitrary configurations, is less than $0.15 \mathrm{~mm}$, it is a good candidate for a model based error correction method.

The GEC calibration method was applied to the PPS with a force/torque sensor built into the system to measure the wrench applied by the patient's weight. It is found that using only 450 calibration measurements the end-point errors could be reduced to well within the required specification. In fact, experimental results show that the maximum error was reduced by a factor of 18 .

\section{ANALYTICAL BACKGROUND}

Physical errors cause the geometric parameters of a manipulator to be different from their ideal values (Roth et al. 1986). As a result, the frames defined at the manipulator joints are slightly displaced from their expected, ideal locations, creating significant end-effector errors. The position and orientation of a frame $\mathrm{F}_{i}^{\text {real }}$ with respect to its ideal location $\mathrm{F}_{i}^{i d e a l}$ is represented by a $4 \times 4$ homogeneous matrix $\mathbf{E}_{i}$. The translational part of matrix $\mathbf{E}_{i}$ is composed of the 3 coordinates $\varepsilon_{x, i}, \varepsilon_{y, i}$ and $\varepsilon_{z, i}$ (along the $\mathrm{X}, \mathrm{Y}$ and $\mathrm{Z}$ axes respectively, defined using the Denavit-Hartenberg representation), see Fig. 3. The rotational part of matrix $\mathbf{E}_{i}$ is the result of the product of three consecutive rotations $\varepsilon_{s, i}, \varepsilon_{r, i}, \varepsilon_{p, i}$ around the $\mathrm{Y}, \mathrm{Z}$ and $\mathrm{X}$ axes respectively (also shown in Fig. $3)$. These are the Euler angles of $\mathrm{F}_{i}^{\text {real }}$ with respect to $\mathrm{F}_{i}^{\text {ideal }}$. The subscripts $\mathrm{s}, \mathrm{r}$, and $\mathrm{p}$ represent spin (yaw), roll, and pitch, respectively. The 6 parameters $\varepsilon_{x, i}, \varepsilon_{y, i}, \varepsilon_{z, i}, \varepsilon_{s, i}, \varepsilon_{r, i}$ and $\varepsilon_{p, i}$ are called generalized error parameters, which can be a function of the system geometry and joint variables. For an $n$ degree of freedom manipulator, there are $6(n+1)$ generalized errors which can be written in the form of a $6(n+1) \times 1$ vector $\varepsilon=\left[\varepsilon_{x, 0}, \ldots, \varepsilon_{x, i}, \varepsilon_{y, i}, \varepsilon_{z, i}, \varepsilon_{s, i}, \varepsilon_{r, i}, \varepsilon_{p, i}, \ldots, \varepsilon_{p, n}\right]$, with $i$ ranging from 0 to $n$, assuming that both the manipulator and the location of its base are being calibrated. The generalized errors that depend on the system geometry, the system task loads and the system joint variables can be calculated from the physical errors link by link. Note that actual system weight effects can be included in the model as a simple function of joint variables.

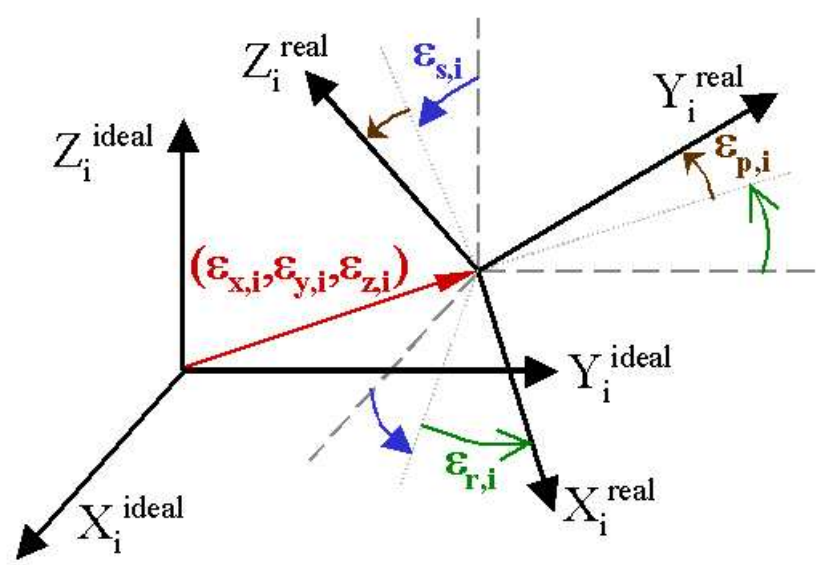

Figure 3: Definition of the Translational and Rotational Generalized Errors for $\mathrm{i}^{\text {th }}$ Link

Since the generalized errors are small, the end-effector position and orientation error $\Delta \mathbf{X}$ can be defined as the $6 \times 1$ vector that represents the difference between the real position and orientation of the end-effector and the ideal one:

$$
\Delta \mathbf{X}=\mathbf{X}^{\text {real }}-\mathbf{X}^{\text {ideal }}
$$

where $\mathbf{X}^{\text {real }}$ and $\mathbf{X}^{\text {ideal }}$ are the $6 \mathrm{x} 1$ vectors composed of the three positions and three orientations of the end-effector reference frame in the inertial reference system for the real and ideal cases, respectively. After linearization, the end-effector error can be represented by the following linear equation:

$$
\Delta \mathbf{X}=\mathbf{J}_{e} \varepsilon
$$

where $\mathbf{J}_{e}$ is the $6 \times 6(n+1)$ Jacobian matrix of the end-effector error $\Delta \mathbf{X}$ with respect to the elements of the generalized error vector $\varepsilon$, also known as Identification Jacobian matrix (Zhuang et al. 1999). As with the generalized errors, $\mathbf{J}_{e}$ depends on the system configuration, geometry and task loads.

If the generalized errors, $\varepsilon \mathfrak{s}$ can be found from calibration measurements, then the correct end-effector position and orientation error can be calculated using Eq. (2) and be compensated. To calculate the generalized errors $\varepsilon$ it is assumed that some components of vector $\Delta \mathbf{X}$ can be measured at a finite number of different manipulator configurations.

Assuming that all 6 components of $\Delta \mathbf{X}$ can be measured, for an $n$ degree of freedom manipulator, $6(n+1)$ generalized errors $\varepsilon$ can be calculated by measuring $\Delta \mathbf{X}$ at $m$ different configurations, defined as $\mathbf{q}_{1}, \mathbf{q}_{2}, \ldots, \mathbf{q}_{m}$, then writing Eq. 
(2) $m$ times:

$$
\Delta X_{t}=\left[\begin{array}{l}
\Delta X_{1} \\
\Delta X_{2} \\
\cdots \\
\Delta X_{m}
\end{array}\right]=\left[\begin{array}{c}
J_{e}\left(q_{1}\right) \\
J_{e}\left(q_{2}\right) \\
\ldots \\
J_{e}\left(q_{m}\right)
\end{array}\right] \cdot \varepsilon=J_{t} \cdot \varepsilon
$$

where $\Delta \mathbf{X}_{t}$ is the $m \times 1$ vector formed by all measured vectors $\Delta \mathbf{X}$ at $m$ different configurations and $\mathbf{J}_{t}$ is the $6 m \mathrm{x}$ $6(n+1)$ matrix formed by the $m$ Identification Jacobian matrices $\mathbf{J}_{e}$ at $m$ configurations, called here Total Identification Jacobian. To compensate for the effects of measurement noise, the number of measurements $m$ is, in general, much larger than $n$.

If the generalized errors $\varepsilon$ are constant, then a unique leastsquares estimate $\hat{e}$ can be calculated (Roth et al. 1986):

$$
\hat{\varepsilon}=\left(J_{t}^{T} J_{t}\right)^{-1} J_{t}^{T} \cdot \Delta X_{t}
$$

However, if the Identification Jacobian matrix $\mathbf{J}_{e}\left(\mathbf{q}_{i}\right)$ contains linearly dependent columns, Eq. (4) will produce estimates with poor accuracy (Hollerbach et al. 1996). This occurs when there is redundancy in the error model, in which case it is not possible to distinguish the error contributed by each generalized error component, even if specific measurement configurations are considered. Orthogonal matrix decomposition can be used in these cases to improve the numerical accuracy of this approach. An analytical method to eliminate the redundant parameters has been presented by (Meggiolaro and Dubowsky 2000). Conventional calibration methods also cannot be successfully applied when some of the generalized errors depend on the manipulator configuration $\mathbf{q}$ or the end-effector wrench $\mathbf{w}$, namely $\varepsilon(\mathbf{q}, \mathbf{w})$, such as when elastic deflections that depend on the configuration and applied forces at the end-effector are significant. A method is presented below for finding the generalized errors $(\varepsilon)$ in the presence of elastic deformations combined with geometric errors.

\section{GEOMETRIC AND ELASTIC ERROR COMPENSATION}

In the GEC method (Geometric and Elastic Error Compensation), elastic deformation and classical geometric errors are considered in a unified manner. The method can identify and compensate for both types of error, without an elastic model of the system. To apply the GEC method, the error model is extended to explicitly consider the task loading wrench and configuration dependency of the errors.

For a system with significant geometric and elastic errors, the generalized errors $\varepsilon$ are a function of the manipulator configuration $\mathbf{q}$ and the end-effector wrench $\mathbf{w}$, or $\varepsilon(\mathbf{q}, \mathbf{w})$. To predict the endpoint position of the manipulator for a given configuration and task wrench, it is necessary to calculate the generalized errors from a set of offline measurements. The complexity of these calculations can be substantially reduced if the generalized errors are parameterized using polynomial functions. The $\mathrm{i}^{\text {th }}$ element of vector $\varepsilon$ is approximated by a polynomial series expansion of the form:

$$
\begin{aligned}
& \varepsilon_{i}^{*}=\sum_{j=1}^{n_{i}} c_{i, j} \cdot f_{i, j}(\mathbf{q}, \mathbf{w}) \\
& f_{i, j}(\mathbf{q}, \mathbf{w}) \equiv\left(w_{m_{j}}\right)^{a_{0, j}} \cdot\left(q_{1}^{a_{1, j}} \cdot q_{2}^{a_{2, j}} \cdot \ldots \cdot q_{n}^{a_{n, j}}\right)
\end{aligned}
$$

where $n_{i}$ is the number of terms used in each expansion, $\mathrm{c}_{i, j}$ are the polynomial coefficients, $\mathrm{w}_{m j}$ is an element of the task wrench $\mathbf{w}$, and $\mathrm{q}_{1}, \mathrm{q}_{2}, \ldots, \mathrm{q}_{n}$ are the manipulator joint parameters. It has been found that good accuracy can be obtained using only a few terms $\mathrm{n}_{i}$ in the above expansion (Meggiolaro et al. 1998). From the definition of the generalized errors, the errors associated with the $i^{t h}$ link depend only on the parameters of the $i^{\text {th }}$ joint. If elastic deflections of link $i$ are considered, then the generalized errors created by these deflections would depend on the weight wrench $\mathrm{w}_{i}$ applied at the $\mathrm{i}^{\text {th }}$ link. For a serial manipulator, this wrench is due to the wrench at the end-effector and to the configuration of the links after the $i^{t h}$. Hence, the wrench $\mathrm{w}_{i}$ depends only on the joint parameters $\mathrm{q}_{i+1}, \ldots, \mathrm{q}_{n}$. Thus, the number of terms in the products of Eq. (5) is substantially reduced. Each generalized error parameter is then represented as a function of only a few of the system variables, greatly reducing the number of measurements required to characterize the system using the GEC method.

The constant coefficients $\mathrm{c}_{i, j}$ are grouped into one vector $\mathbf{c}$, becoming the unknowns of the problem. The total number of unknown coefficients, called $n_{c}$, is the sum of the number of terms used in Eq. (5) to approximate each generalized error, i.e. $n_{c}=\Sigma n_{i}$. The $n_{c}$ functions $\mathrm{f}_{i, j}(\mathbf{q}, \mathbf{w})$ are then incorporated into the Identification Jacobian matrix $\mathbf{J}_{e}$ from Eq. (2):

$$
\Delta \mathbf{X}=\mathbf{J}_{e}(\mathbf{q}) \cdot \varepsilon(\mathbf{q}, \mathbf{w}) \equiv \mathbf{H}_{e}(\mathbf{q}, \mathbf{w}) \cdot \mathbf{c}
$$

where $\mathbf{H}_{e}$ is the (6 $\left.\mathrm{x} n_{c}\right)$ Jacobian matrix of the end-effector error $\Delta \mathbf{X}$ with respect to the polynomial coefficients $\mathrm{c}_{i, j}$. The matrix $\mathbf{H}_{e}$, called here Extended Identification Jacobian matrix, can be obtained from Eqs. (5) and (6):

$$
\begin{aligned}
\mathbf{H}_{e}(\mathbf{q}, \mathbf{w}) \equiv\left[\mathbf{J}_{1} \cdot f_{1,1}, \ldots, \mathbf{J}_{1} \cdot f_{1, n 1}, \ldots\right. \\
\\
\left.\quad \mathbf{J}_{i} \cdot f_{i, 1}, \mathbf{J}_{i} \cdot f_{i, 2}, \ldots, \mathbf{J}_{i} \cdot f_{i, n i}, \ldots\right]
\end{aligned}
$$

where $\mathbf{J}_{i}$ is the column of matrix $\mathbf{J}_{e}$ associated to the generalized error component $\varepsilon_{i}$.

An estimate of the coefficient vector $\mathbf{c}$ is then calculated by replacing $\mathbf{J}_{e}$ with the matrix $\mathbf{H}_{e}$ in Eq. (3) and applying 
Eq. (4), completing the identification process, see Eq. (8). Once the constant polynomial coefficients, c, are identified, the end-effector position and orientation error $\Delta \mathbf{X}$ can be calculated and compensated using Eq. (6).

$$
\hat{\mathbf{c}}=\left(\mathbf{H}_{\mathbf{e}}^{\mathbf{T}} \mathbf{H}_{\mathbf{e}}\right)^{-1} \mathbf{H}_{\mathbf{e}}^{\mathbf{T}} \cdot \Delta \mathbf{X}
$$

Finally, it must be emphasized that the GEC model has the advantage of modeling non-linear elasticity, due to its polynomial nature. The polynomial approximation would only be a model of linear elasticity if the order of the polynomial was limited to three (relating the joint parameters, or order one relating the payload wrench), and if these polynomial coefficients were related among themselves in the same way as in the analytical results for simple beam bending. However, the polynomial expansion can include additional terms, being able to model general non-linear (or linear) elasticity, combined with a general formulation for geometric errors that may vary in their own frames (and thus not limited to constant geometric errors).

\section{APPLICATION TO THE PATIENT POSI- TIONING SYSTEM}

The PPS is a six degree of freedom robot manipulator (see Fig. 2) built by General Atomics (Flanz 1996). The first three joints are prismatic, with maximum travel of $225 \mathrm{~cm}$, $56 \mathrm{~cm}$ and $147 \mathrm{~cm}$ for the lateral (X), vertical (Y) and longitudinal $(Z)$ axes, respectively. The last three joints are revolute joints. The first joint rotates parallel to the vertical (Y) axis and can rotate $\pm 90^{\circ}$. The last two joints are used for small corrections around an axis of rotation parallel to the $\mathrm{Z}$ (roll) and $X$ (pitch) axes, and have a maximum rotation angle of $\pm 3^{\circ}$. The manipulator "end-effector"is a couch, supporting the patient in a supine position, accommodating patients up to $188 \mathrm{~cm}$ in height and $300 \mathrm{lbs}$ in weight in normal operation.

The intersection point of the proton beam with the gantry axis of rotation is called the system isocenter. The treatment volume is defined by a treatment area on the couch of $50 \mathrm{~cm} \mathrm{x}$ $50 \mathrm{~cm}$ and a height of $40 \mathrm{~cm}$ (see Figure 2). This area covers all possible locations of treatment points (i.e. tumor locations at a patient). The objective is that the PPS makes any point in this volume be coincident with the isocenter at any orientation.

The joint parameters of the PPS are the displacements $d_{1}$, $\mathrm{d}_{2}, \mathrm{~d}_{3}$ of the three prismatic joints and the rotations $\theta, \alpha, \beta$ of the three rotational joints. A 6 axis force/torque sensor is placed between the couch and the last joint. By measuring the forces and moment at this point, it is possible to calculate the patient weight and the coordinates of the patient center of gravity. The system motions are very slow and smooth due to safety requirements. Hence, the system is quasi-static, and its dynamics do not influence the system accuracy and are neglected.

The accuracy of the PPS was measured with a position accuracy of approximately $0.04 \mathrm{~mm}$ using a Leica 3D Laser Tracking System. These measurements were to evaluate the PPS repeatability, the nonlinearity of its weight-dependent deflections, the inherent uncompensated PPS accuracy, and the method developed above.

Three targets were placed about $10 \mathrm{~mm}$ above the couch. For more than 700 different configurations of the PPS and different weights the location of the three targets is measured. From the system kinematic model with no errors, the ideal coordinates of the Nominal Treatment Point (NTP), defined as the location of a tumor on a patient, were calculated and subtracted from the experimentally measured values to yield the vector $\Delta \mathbf{X}(\mathbf{q}, \mathbf{w})$. Then, 450 measurements were used to evaluate the basic uncompensated accuracy of the PPS and the accuracy of the compensation method described above. Two different payloads were used: one with no weight and another with a $70 \mathrm{~kg}$ weight at the center of the treatment area. The PPS configurations used were grouped into two sets:

Set a) Treatment Volume. The 8 vertices of the treatment volume (see Figure 2) are reached with the NTP with angle $\theta$ taking values from $-90^{\circ}$ to $90^{\circ}$ with a step of $30^{\circ}$, for a total of 112 configurations.

Set b) Independent Motion of Each Axis. Each axis is moved independently while all other axes are held at the home (zero) values. The step of motion for $d_{1}$ is $50 \mathrm{~mm}$, for $d_{2} 20 \mathrm{~mm}$, for $\mathrm{d}_{3} 25 \mathrm{~mm}$ and for $\theta 5^{\circ}$, resulting in 338 configurations.

The PPS uncompensated accuracy combining the two sets is shown in Figure 4. The data points represent the positioning errors of NTP. It is clearly seen that in spite of the high quality of the PPS physical system, its uncompensated accuracy is on the order of $10 \mathrm{~mm}$. This is approximately 20 times higher than the specification of $\pm 0.50 \mathrm{~mm}$.

Part of the uncompensated error is the repeatability errors. These errors are due to the random system errors, and they cannot be compensated by the GEC method. They represent the accuracy limit of any error compensation algorithm and it also shows how well an error compensation technique performs. Here the system repeatability is based on how well the system would return the NTP to certain arbitrary configurations. A total of 270 measurements were taken with zero payload weight. The repeatability error of the PPS is less than $0.15 \mathrm{~mm}(3 \sigma)$. Thus this system is a good candidate for model based error correction methods, since the repeatability errors are relatively small compared to the $\pm 0.50 \mathrm{~mm}$. 




Figure 4: Measured and Residual Errors After Compensation

In implementing the computation method a general nonlinear function of the wrench $\mathbf{w}$ can be used. To help establish this function, the behavior of the PPS positioning errors for different payload weights was examined with measurements made at the home (zero) configuration. The weights ranged from 0 to $300 \mathrm{lbs}$ in steps of approximately $25 \mathrm{lbs}$. The results showed that the positioning errors of the PPS are nearly linear with the payload weight. The least square error is less than $0.1 \mathrm{~mm}$ for the linear fit. Hence the generalized errors were taken as linear functions of the system wrench in Eq. (5).

The generalized errors are then calculated with Eq. (4) using the configurations of set (b) (independent motion of its axes) and half of the treatment volume data (set a). For a Pentium PC $300 \mathrm{MHz}$, the computing time was less than one minute. The PPS is then commanded to go to compensated points for the remaining configurations of set (a). The residual positioning errors of the PPS after compensation for these points are shown in Fig. 4. The residual errors are enclosed in a sphere of $0.38 \mathrm{~mm}$ radius which is smaller than the accuracy specification. The required number of data points for this calculation was less than 400 . Hence the compensation method used in this paper enables the system to meet its specification. It is now a key element in MGH's operational software. Since the remaining errors after calibration using 400 points were comfortably under $0.5 \mathrm{~mm}$, a significantly smaller number of poses could have been used in the calibration. In fact, applying the presented calibration method to a subset of only 125 measurement poses of the Patient Positioning System resulted in a maximum residual error of $0.49 \mathrm{~mm}$. This absolute accuracy meets the specification, while significantly less than 400 measurement points were necessary. This number is indeed much smaller than it might be expected, considering that not only elastic deformations, but also geometric errors that vary in their own frames (such as a quasi-sinusoidal shape for the rail errors on the prismatic base, as discussed below), are present in the system. However, the calibration error dependence on the number of measurement poses has not been addressed in this work.

One of the main advantages of the GEC methodology is the ability to model non-linearities or any other repeatable error source that can be represented as a function of the system parameters and of the payload wrenches. Since any differentiable analytical expression can be represented as a polynomial series, the method is able to identify errors that other calibration methods (which only model elastic deformations and geometric errors constant in their frames) can't. In particular, the errors along the Patient Positioner's lateral rail had an approximately sinusoidal shape (as it was expected from the respective manufacturing process, due to eccentricities in its machining), which turned out to be an important error source in this system. These errors were identified through the presented methodology using polynomial expansions with relatively few terms (about 8th order).

In addition, the GEC method has an advantage of automatically accounting for the elastic deformations due to link masses. The polynomial terms that are a function of the system configuration (but not of the task wrench w) are the ones that account for the contribution of the link masses to the varying end-effector elastic errors. Since the link masses are constant (only the associated moments are variable), the constant polynomial coefficients associated with these terms will automatically account for these masses. These errors are clearly configuration-dependent, as expected, since the polynomial terms that multiply these constant mass coefficients are a function of the system configuration. Therefore, all link masses are implicitly identified, and their associated elastic errors are automatically compensated for.

\section{CONCLUSIONS}

In this work, a method is discussed to compensate the positioning end-effector errors of large manipulators with significant task loads. Both geometric and elastic errors are considered without requiring an explicit elastic model of the system. The method has been applied experimentally to a highaccuracy large medical manipulator. The results showed that the basic accuracy of the manipulator exceeded its specifications, but after applying the method to compensate for end-effector errors the accuracy specifications are met. The method is now a key element of the software used to treat cancer patients.

\section{ACKNOWLEDGEMENTS}

The support of the National Institutes of Health via the Northeast Proton Therapy Center from the Massachusetts General Hospital for this work are acknowledged. The contribution of P. Drouet is gratefully acknowledged. 


\section{REFERENCES}

Drouet, P., Dubowsky, S. and Mavroidis, C. (1998). Compensation of Geometric and Elastic Deflection Errors in Large Manipulators Based on Experimental Measurements, Proceedings of the 6th Int. Symposium on Advances in Robot Kinematics, Austria, pp. 513-522.

Flanz, J. (1996). Design Approach for a Highly Accurate Patient Positioning System for NPTC, Proceedings of the PTOOG XXV and Hadrontherapy Symposium, Belgium, pp. 1-5.

Hamel W., Marland S. and Widner T. (1997). A ModelBased Concept for Telerobotic Control of Decontamination and Dismantlement Tasks, Proceedings of the 1997 IEEE International Conference of Robotics and Automation, Albuquerque, New Mexico.

Hollerbach, J.M., Wampler, C.W. (1996). The Calibration Index and Taxonomy for Robot Kinematic Calibration Methods, International Journal of Robotics Research, Vol. 15, No. 6, pp. 573-591.

Mavroidis, C., Dubowsky, S., Drouet, P., Hintersteiner, J., Flanz, J. (1997). A Systematic Error Analysis of Robotic Manipulators: Application to a High Performance Medical Robot, Proceedings of the 1997 IEEE Int. Conference of Robotics and Automation, Albuquerque, New Mexico, pp. 980-985.

Meggiolaro, M., Mavroidis, C., Dubowsky, S. (1998). Identification and Compensation of Geometric and Elastic Errors in Large Manipulators: Application to a High Accuracy Medical Robot, Proceedings of the 25th Biennial Mechanisms Conference, ASME, Atlanta.

Meggiolaro, M., Dubowsky, S. (2000). An Analytical Method to Eliminate the Redundant PaRAMETERS IN RoBot CALIBRATION, PROCEEDINGS OF THE International Conference on Robotics and Automation (ICRA '2000), IEEE, SAN FRANCISCO, PP. 3609-3615.

Rabinowitz, I. (1985). Accuracy of Radiation Field Alignment in Clinical Practice, International Journal of Radiation Oncology, Biology and Physics, Vol.11, pp.18571867.

Roth, Z.S., Mooring, B.W., Ravani, B. (1986). An Overview of Robot Calibration, IEEE Southcon Conference, Orlando, Florida, pp. 377-384.

Vaillancourt C. and Gosselin, G. (1994). Compensating for the Structural Flexibility of the SSRMS with the SPDM, Proceedings of the International Advanced Robotics Program, Second Workshop on Robotics in Space, Canadian Space Agency, Montreal, Canada.
Zhuang, H., Motaghedi, S.H., Roth, Z.S. (1999). Robot Calibration with Planar Constraints, Proc. IEEE International Conference of Robotics and Automation, Detroit, Michigan, pp.805-810. 